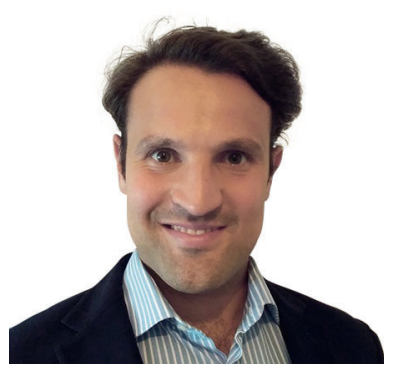

\title{
Diabetisches Makulaödem: Strukturelle und funktionelle Ergebnisse der sequentiellen Dexamethason- und Aflibercept- Therapie nach einem Jahr Behandlung
}

\section{Emiliano Di Carlo}

Augenklinik, Städtische Klinikum Karlsruhe, Karlsruhe, Deutschland

Abstract aus Hernández-Bel L, Cervera-Taulet E, Navarro-Palop C, et al.: Sequential desamethasone and Aflibercept treatment in patients with diabetic macular edema: structural and functional outcomes at 52 weeks. Ophthalmologica 2019;241:98-104.

\section{Keywords}

Diabetic macular edema • Aflibercept • Dexamethasone implant

\begin{abstract}
Objective: The aim of this paper is to compare intravitreous aflibercept versus dexamethasone implant followed by aflibercept (sequential treatment group) in patients with diabetic macular edema (DME).
\end{abstract}

Methods: We conducted an observational retrospective study in naive DME patients, 15 treated only with aflibercept (a monthly injection for the first 5 consecutive doses, followed by an injection every 2 months), and 15 treated with a single dexamethasone implant followed by bimonthly aflibercept. Best-corrected visual acuity (BCVA), central macular thickness (CMT), and qualitative features as well as adverse events were assessed at baseline and at 2, 6, and 12 months.
Results: BCVA increased from $70.8 \pm 4.1$ to $83.5 \pm 2.7$ letters with aflibercept and from $75.6 \pm 2.7$ to $86.5 \pm 2.5$ with sequential treatment ( $p=0.551)$. CMT decreased from $411 \pm 26.1$ to $288.1 \pm 10.5$ with aflibercept and from $411.4 \pm 24.3$ to $260.8 \pm 17.9$ in the sequential treatment group. The differences between the 2 groups, in terms of visual gain and decreased MT, were not statistically significant $(p>0.05)$. Nine and 6 injections and 9 and 7 monitoring visits were performed.

Conclusion: Sequential treatment in DME, starting with dexamethasone and followed by aflibercept, is a promising alternative that can reduce the treatment burden in the first year without statistically significant differences in terms of visual gain and decreased MT compared to aflibercept only.

(c) 2018 S.Karger AG, Basel

\section{KARGER}

() 2019 S. Karger GmbH, Freiburg

Fax +497614520714 


\section{Transfer in die Praxis}

\section{Hintergrund}

Das diabetische Makulaödem (DMÖ) ist die häufigste Erblindungsursache bei Patienten mit Diabetes mellitus, mit einer Prävalenz von 7\% [1]. In den letzten Jahren wurden zwei grundlegend verschiedene Therapiestrategien, die Behandlung mit Anti-VEGF und die Behandlung mit Kortikosteroiden, intensiv untersucht.

Mehrere randomisierte klinische Studien haben den Nutzen der Gabe von Anti-VEGF-Medikamenten sowohl im Bezug auf die bestkorrigierte Sehschärfe (BCVA) als auch auf die Reduktion der zentralen Netzhautdicke (CRT) nachgewiesen [2-4].

Das intravitreale Dexamethason-Implantat wurde im Juli 2014 von der Europäischen Arzneimittel-Agentur (EMA) für die Behandlung von Patienten mit Sehschärfenreduktion infolge eines DMÖ zugelassen [5].

Ziel der hier diskutierten Studie war es, die Sehschärfenergebnisse von behandlungsnaiven DMÖ-Patienten zu untersuchen. Man hat zwei Therapieansätze verglichen: intravitreales Dexamethasonimplantat, gefolgt von monatlichen Injektionen von Aflibercept vs. Behandlung mit einem standardisierten Aflibercept-Protokoll.
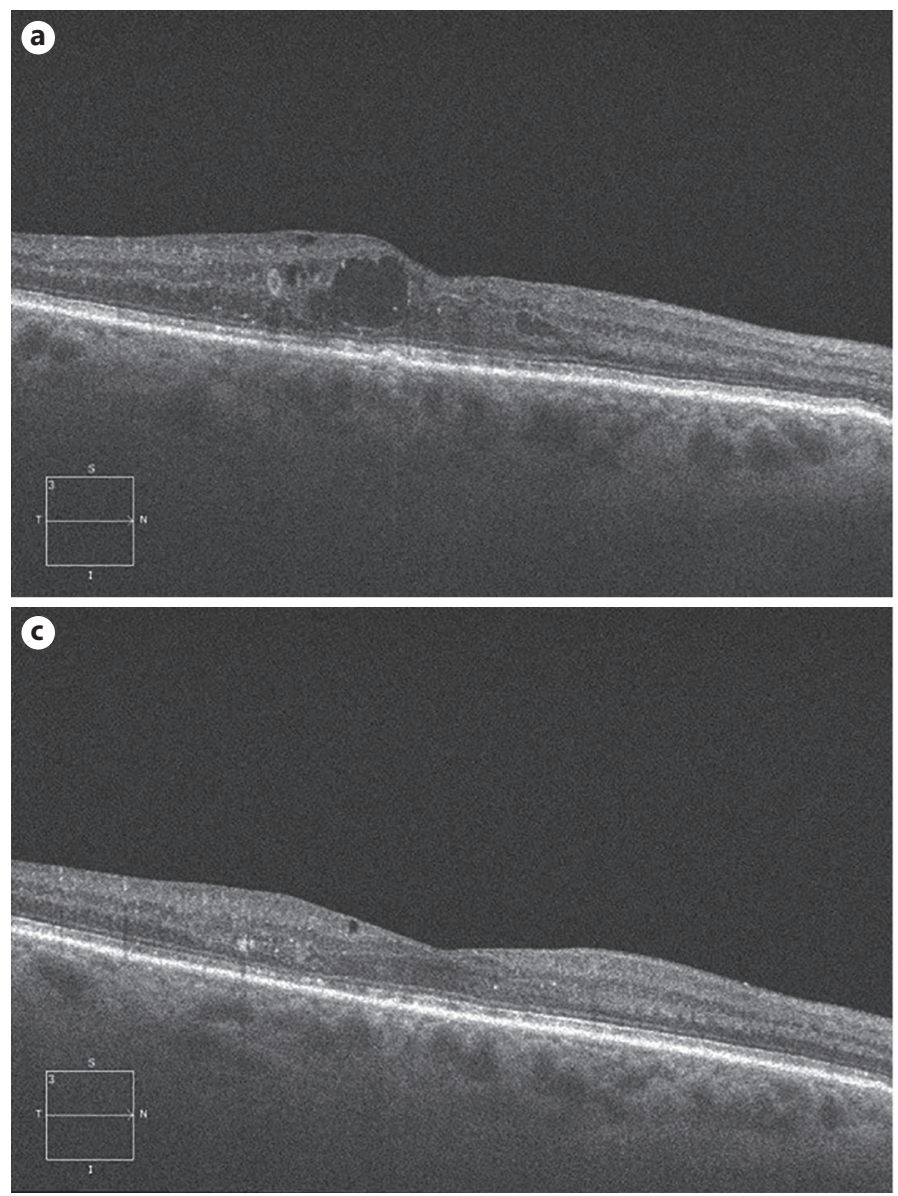

\section{Methoden und Ergebnisse}

Es handelt sich hier um eine retrospektive Beobachtungsstudie von Patienten mit der Erstdiagnose DMÖ, die innerhalb eines Jahres behandelt wurden (Follow-up-Dauer: 52 Wochen).

30 Patienten wurden in zwei Gruppen von je 15 Patienten eingeteilt. Gruppe 1 erhielt ein standardisiertes Therapieprotokoll (5 monatliche Injektionen Aflibercept, gefolgt von einer Injektion alle $2 \mathrm{Mo}$ nate). Gruppe 2 wurde mit einem Dexamethason-0,7-mg-Implantat behandelt, gefolgt von zweimonatlichen intravitrealen Aflibercept-Injektionen ab der 16. Woche.

BCVA und CRT wurden am Ende der Behandlung (52 Wochen) zwischen den zwei Gruppen verglichen. Es wurde kein statistisch signifikanter Unterschied beobachtet.

\section{Kritik}

Die optimale Behandlung des DMÖ stellt eine klinisch relevante Herausforderung dar. Verschiedene Studien deuten darauf hin, dass die Kombinationsbehandlung bestehend aus Anti-VEGF und intravitrealer Steroidgabe eine frühere Visusverbesserung und eine längere Wirkungsdauer bewerkstelligen kann [6-7]. In diesem Zusammenhang bietet die sequentielle Therapie die Möglichkeit, die Wirkungen einer Anti-VEGF-Therapie, wie z.B. die Hemmung der Gefäßpermeabilität

Abb. 1. Unsere Erfahrung mit einer kombinierten (sequenziellen) Dexamethason- und Aflibercept-Therapie: SD-OCT-Untersuchungen (Beginn der Behandlung, 2 Monate, 6 Monate, 12 Monate). Resolution der SRF und Verbesserung der intraretinalen Zysten.

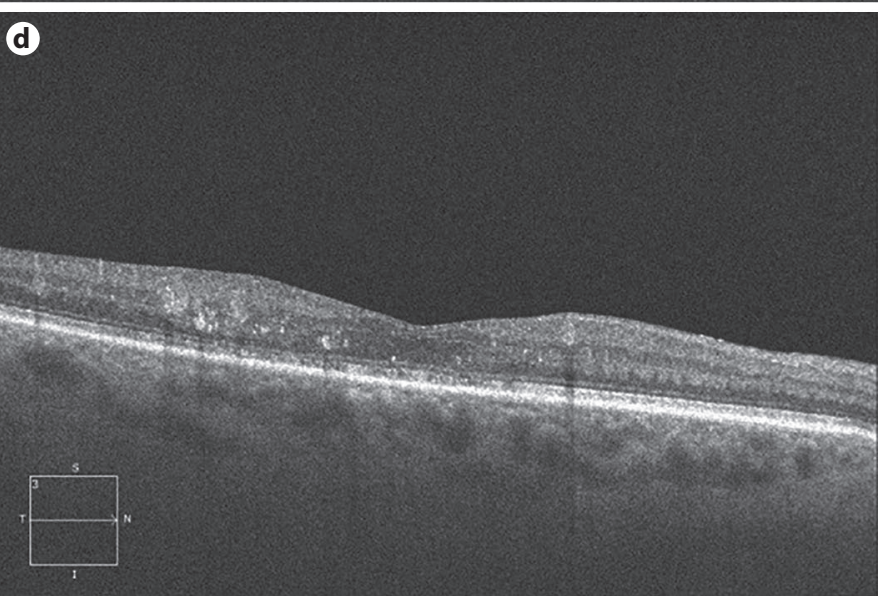


und der Angiogenese, mit der entzündungshemmenden Wirkung des Cortisons (Stabilisierung der Blut-Retina-Schranke, die Regulierung der Aktivität der Müllerzellen u.a.) zu kombinieren. So kann man die Vorteile beider Therapiestrategien nutzen.

Die Notwendigkeit der häufigen Wiederbehandlung bei AntiVEGF-Monotherapie hat trotz der positiven Wirkungen zahlreiche Nachteile, die letztlich die Vorteile dieser Strategie oft minimieren: Insbesondere die hohe Belastung für die Patienten und ihre Angehörigen sowie die Kosten für das Gesundheitssystem müssen hier erwähnt werden. Ersteres führt of zu einer reduzierten Compliance, was wiederum die Ergebnisse schmälert.

Aus diesem Grund kann die Standardisierung einer sequentiellen Behandlung, beginnend mit einem Dexamethasonimplantat und gefolgt von Aflibercept-Injektionen, die Behandlungsbelastung vor allem im ersten Jahr reduzieren, indem sie die Anzahl der Arztbesuche und auch die Kosten für das Gesundheitssystem reduziert (Abb. 1).

\section{Fazit}

Die sequentielle Behandlungsmodalität, die aus DexamethasonImplantat und Aflibercept besteht, stellt eine wichtige alternative Behandlungsmethode bei DMÖ-Patienten dar. In der klinischen Praxis werden die stringenten Behandlungsintervalle, die bei AntiVEGF-Monotherapie notwendig sind, oft nicht eingehalten, da die Patientencompliance sinkt und so die schließlich notwendigen Behandlungskosten sogar noch ansteigen können.

Daher ermöglicht die Kombination einer sequentiellen Dexamethason Behandlung mit Aflibercept sowohl den Ärzten als auch den Patienten ein gutes Werkzeug zur Behandlung dieser chronischen Erkrankung, die eine jahrelange kontinuierliche Therapie erfordert.

\section{Disclosure Statement}

Hiermit erkläre ich, dass keine Interessenskonflikte in Bezug auf den vorliegenden Kommentar bestehen.

\section{Literatur}

1 Yau JW, Rogers SL, Kawasaki R, et al.; Meta-Analysis for Eye Disease (METAEYE) Study Group. Global prevalence and major risk factors of diabetic retinopathy. Diabetes Care 2012; 35: 556-564.

2 Cheung N, Mitchell P, Wong TY: Diabetic retinopathy. Lancet 2010;376: 124-136.

3 Diabetic Retinopathy Clinical Research Network, Elman MJ, Aiello LP, et al.: DRCR.net. Protocol l: randomized trial evaluating ranibizumab plus prompt or deferred laser or triamcinolone plus prompt laser for diabetic macular edema. Ophthalmology 2010;117:1064-1077.

4 Elman MJ, Bressler NM, Qin H, et al.: Diabetic Retinopathy Clinical Research Network. Expanded 2-year follow-up of ranibizumab plus prompt or deferred laser or triamcinolone plus prompt laser for diabetic macular edema. Ophthalmology 2011;118:609-614.

5 Schmidt-Erfurth U, Garcia-Arumi J, Bandello F, et al.: guidelines for the management of diabetic macular edema by the European Society of Retina Specialists (EURETINA). Ophthalmologica 2017;237:185-222.

6 Faghihi H, Roohipoor R, Mohammadi SF, et al.: Intravitreal bevacizumab versus combined bevacizumab-triamcinolone versus macular laser photocoagulation in diabetic macular edema. Eur J Ophthalmol 2008;18: 941-948.

7 Ahmadieh H, Ramezani A, Shoeibi N, et al.: Intravitreal bevacizumab with or without triamcinolone for refractory diabetic macular edema; a placebo controlled, randomized clinical trial. Graefes Arch Clin Exp Ophthalmol 2008;246:483-489.

Kontaktadresse: Dr. Emiliano Di Carlo, Augenklinik, Städtisches Klinikum Karlsruhe, Moltkestraße 90, 76133 Karlsruhe, Deutschland,

emi.dicarlo@hotmail.it 